\title{
Strong Edge Cover of the Graph
}

\author{
D. K. Thakkar ${ }^{1}$, B. M. Kakrecha ${ }^{2}$ \\ 1 Department of Mathematics, Saurashtra University, Gujarat, India \\ 2 Department of Mathematics, L. E. College, Gujarat, India.
}

*Corresponding Author: D. K. Thakkar, Department of Mathematics, Saurashtra University, Gujarat, India

\begin{abstract}
The paper is about strong edge cover of the graph. The edge set of the graph is a strong edge cover if all the pendent edges belong to the edge set and among the non pendent edges if two distinct edges are adjacent then one edge must belong to the edge set. It is proved that every strong edge cover is an edge cover but the converse need not be true. The characterization of a minimal strong edge cover is given. The relation between strong edge cover and edge H-domination is discussed. The effect of vertex (edge) removal operation on strong edge covering number of the graph is observed.
\end{abstract}

Keywords: edge cover, edge H-dominating set, strong edge cover

AMS Subject Classification: (2010) - 05C69.

\section{INTRODUCTION}

Let $\mathrm{G}=(\mathrm{V}, \mathrm{E})$ be a finite, simple, undirected graph. The vertex set of $\mathrm{G}$ is denoted by $\mathrm{V}(\mathrm{G})$ (or simply V) and edge set by $\mathrm{E}(\mathrm{G})$ (or $\mathrm{E}$ ). Each edge $\mathrm{e} \in \mathrm{E}$ is an unordered pair of distinct vertices of $\mathrm{V}$. If an edge $\mathrm{e}=\mathrm{uv}$ then we say that a vertex $\mathrm{u}$ is adjacent to vertex $\mathrm{v}$ or the vertices $\mathrm{u}$ and $\mathrm{v}$ are neighbors and that $\mathrm{e}$ is incident to $\mathrm{u}$ and $\mathrm{v}$.

A subgraph $H$ of $G$ is a graph such that $V(H) \subseteq V(G)$ and $E(H) \subseteq E(G)$. For a vertex $v \in V(G)$, the graph $G-v$ is called a vertex-deleted subgraph of $G$. If $v u \in E(G)$ then $G-v u$ is called an edge-deleted subgraph of $\mathrm{G}$.

The degree of a vertex $\mathrm{v}($ denoted by $\operatorname{deg}(\mathrm{v}))$ is equal to the number of vertices that are adjacent to $v$. If there is a vertex $v \in V(G)$ such that $\operatorname{deg}(v)=0$ then $v$ is called an isolated vertex. If $\operatorname{deg}(v)=$ 1 then $\mathrm{v}$ is called a pendent vertex. An edge $\mathrm{e}=\mathrm{uv}$ is an isolated edge of $\mathrm{G}$ if $\operatorname{deg}(\mathrm{u})=1$ and $\operatorname{deg}(\mathrm{v})=1$ in $\mathrm{G}$ also $\mathrm{e}=\mathrm{uv}$ is a pendent edge of $\mathrm{G}$ if the degree of exactly one of $\mathrm{u}$ and $\mathrm{v}$ is 1 in $\mathrm{G}$. The minimum degree (respectively, the maximum degree) of the graph $\mathrm{G}$ is denoted by $\delta(\mathrm{G})$ (respectively, $\Delta(\mathrm{G})$ ).

The concept of vertex cover is well-known [1,2]. A vertex covering set $\mathrm{S} \subseteq \mathrm{V}$ of $\mathrm{G}$ is a set of vertices such that every edge of $G$ has at least one end vertex in $S$. The vertex covering number (denoted by $\alpha_{0}(\mathrm{G})$ ) of $\mathrm{G}$ is the minimum cardinality of a vertex covering set of $\mathrm{G}$ [2].

Here, a concept known as an edge cover of the graph is considered and a new concept called strong edge cover of the graph is defined. The relation between edge H-domination and strong edge cover is discussed. The effect of vertex(edge) removal on the strong edge covering number is observed.

Definition 1.1. [2] (edge cover) A subset $F$ of $E(G)$ is said to be an edge cover of the graph $G$ if every vertex of $G$ is an end vertex of some edge of $F$.

Definition 1.2. [3] (strong edge cover) An edge set $F$ of the graph $G$ is said to be a strong edge cover of $\mathrm{G}$ if the following conditions are satisfied.

(1) If $e$ is an edge such that at least one end vertex of $e$ is a pendant vertex then $e \in F$.

(2) If $e_{1}$ and $e_{2}$ are distinct edges of $G$ such that $e_{1}$ and $e_{2}$ are adjacent then $e_{1} \in F$ or $e_{2} \in F$.

In this paper, we consider only those graphs which do not contain isolated vertices. 


\section{Relation between Edge Cover AND STRONG EDGe Cover}

Example 2.1. Consider the complete graph $\mathrm{K}_{4}$ with vertices $1,2,3,4$ and edges $12,23,34,41,13$, 24.

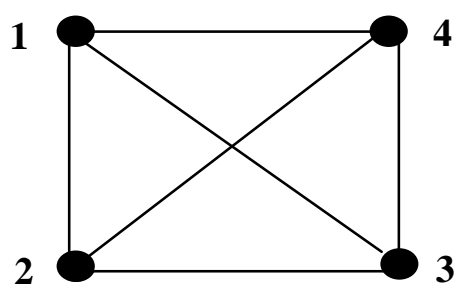

Figure 1. Complete graph $\mathrm{K}_{4}$

The edge set $F=\{13,24\}$ is an edge cover of $K_{4}$ but $F$ is not a strong edge cover of $\mathrm{K}_{4}$ because 12 and 14 are distinct adjacent edges of $\mathrm{K}_{4}$ and none of 12 and 14 belongs to $\mathrm{F}$.

Proposition 2.2. Every strong edge cover of the graph is an edge cover.

Proof. Let $F$ be a strong edge cover of the graph $G$ and $v$ be any vertex of $G$. Suppose that $v$ is a pendant vertex then the edge e incident to the vertex $v$ must be in $F$. Suppose that $v$ is not a pendant vertex then $\operatorname{deg}(\mathrm{v}) \geq 2$ and there are at least 2 edges say e and $\mathrm{f}$ incident at $\mathrm{v}$. Since $\mathrm{F}$ is a strong edge cover of $G$, either $e \in F$ or $f \in F$. If $e \in F$ then $e$ is an edge of $F$ which contains $v$ and if $f \in F$ then $f$ is an edge of $F$ which contains $v$. Thus $F$ is an edge cover of $G$.

Proposition 2.3. An edge cover of the graph need not be a strong edge cover.

Definition 2.4. [2] An edge cover with minimum cardinality is a minimum edge cover of G. The cardinality of a minimum edge cover is called the edge covering number of $G$ and it is denoted by $\alpha_{1}(\mathrm{G})$.

Definition 2.5. [3] A strong edge cover of the graph $\mathrm{G}$ with minimum cardinality is called a minimum strong edge cover. The cardinality of a minimum strong edge cover is the strong edge covering number of $\mathrm{G}$, denoted by $\alpha_{1}^{S}(\mathrm{G})$.

Remark 2.6. (1) Every strong edge cover of the graph $G$ with $\Delta(G) \leq 2$ is an edge cover. That is every strong edge cover of a path graph and a cycle graph is an edge cover.

(2) By proposition 2.2, $\alpha_{1}(\mathrm{G}) \leq \alpha_{1}^{S}(\mathrm{G})$.

\section{Relation between EDGe H-DOMination AND STRONG EDGe COVER}

Definition 3.1. $[3,5]$ (edge $H$-dominating set) $A$ set $F \subseteq E(G)$ is said to be an edge

$H$-dominating set of $G$ if the following conditions are satisfied by any edge $e=u v$ in $E(G)$.

(1) If $e$ is an isolated edge then $e \in F$.

(2) If $\mathrm{e}$ is a pendant edge with $\mathrm{v}$ as a pendant vertex and $\mathrm{u}$ is not a pendant vertex. If $\mathrm{uv} \notin \mathrm{F}$ then all the edges incident at $\mathrm{u}$ (except $\mathrm{e}$ ) are in $\mathrm{F}$.

(3) If $\mathrm{e}$ is a pendant edge with $\mathrm{u}$ as a pendant vertex and $\mathrm{v}$ is not a pendant vertex. If $\mathrm{uv} \notin \mathrm{F}$ then all the edges incident at $\mathrm{v}$ (except $\mathrm{e}$ ) are in $\mathrm{F}$.

(4) If neither $u$ nor $v$ is a pendant vertex and $u v \notin F$ then all the edges incident at $u$ (except e) are in $\mathrm{F}$ or all the edges incident at $\mathrm{v}$ (except $\mathrm{e}$ ) are in $\mathrm{F}$.

Example 3.2. Consider the following graph with vertices 1, 2, 3, 4 and edges 12, 23, 34, 41, 13.

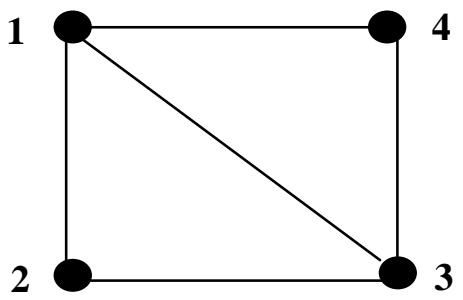

Figure 2. Graph G with 4 vertices 
The edge set $F=\{23,34\}$ is an edge $\mathrm{H}$-dominating set of the graph $\mathrm{G}$ but $\mathrm{F}$ is not a strong edge cover of $\mathrm{G}$ because 12 and 14 are distinct adjacent edges of $\mathrm{G}$ and none of 12 and 14 belongs to $\mathrm{F}$.

Proposition 3.3. Every strong edge cover is an edge H-dominating set.

Proof. Let $\mathrm{F}$ be a strong edge cover. Let e be an edge such that e $\notin F$. Since F contains all isolated edges, e cannot be an isolated edge. Let $e=u v$ and let $f$ be any edge incident at $u$ then $e$ and $f$ are adjacent edges. Since e $\notin F$ and $F$ is a strong edge cover $f \in F$. Therefore all the edges incidentat $u$ (except e) are in F. Similarly, all the edges incident at v (except e) are in F. This proves that $\mathrm{F}$ is an edge H-dominating set.

Proposition 3.4. An edge H-dominating set need not be a strong edge cover.

Definition 3.5. (minimal strong edge cover) A strong edge cover $F$ of the graph $G$ is said to be a minimal strong edge cover if for every edge e $\in \mathrm{F}, \mathrm{F}-\{\mathrm{e}\}$ is not a strong edge cover of $\mathrm{G}$.

Example 3.6. Consider the graph given in figure 2, the strong edge cover $\{12,13,34\}$ is a minimal strong edge cover.

The characterization of a minimal strong edge cover can be stated as follows.

Theorem 3.7. Let $G$ be a graph and $F$ be a strong edge cover of $G$. $F$ is a minimal strong edge cover if and only if for every edge $e \in F$, one of the following two conditions holds.

(1) e has one end vertex which is a pendant vertex.

(2) There is an edge $\mathrm{g}$ adjacent to e such that $\mathrm{g} \notin \mathrm{F}$.

Proof. Suppose that $\mathrm{F}$ is a minimal strong edge cover of the graph G. Therefore for any edge e $\in$ $\mathrm{E}(\mathrm{G}), \mathrm{F}-\{\mathrm{e}\}$ is not a strong edge cover of $\mathrm{G}$. Therefore one of the following two possibilities holds.

(1) There is an edge $f \in E(G)$ such that one end vertex of $f$ is a pendant vertex but $f \notin F-\{e\}$. Since $F$ is a strong edge cover of $G, f \in F$ and therefore $f=e$. Thus, condition (1) is satisfied.

(2) There are two adjacent edges $\mathrm{g}$ and $\mathrm{h}$ but $\mathrm{g} \notin \mathrm{F}-\{\mathrm{e}\}$ and $\mathrm{h} \notin \mathrm{F}-\{\mathrm{e}\}$. Since $\mathrm{F}$ is a strong edge cover of $\mathrm{G}, \mathrm{g} \in \mathrm{F}$ or $\mathrm{h} \in \mathrm{F}$. This implies that one of the edges $\mathrm{g}$ and $\mathrm{h}$ does not belong to $\mathrm{F}$ and the other edge is equal to e. We may assume that $\mathrm{h}=\mathrm{e}$ then $\mathrm{g}$ is adjacent with $\mathrm{e}$ and $\mathrm{g} \notin \mathrm{F}$. Thus, condition (2) is satisfied.

Conversely, Suppose that condition (1) or condition (2) holds. Let e $\in$ F. If condition (1) holds then $\mathrm{e} \notin \mathrm{F}-\{\mathrm{e}\}$ it implies that $\mathrm{F}-\{\mathrm{e}\}$ is not a strong edge cover of $\mathrm{G}$. If condition (2) holds then there is an edge $\mathrm{g}$ such that $\mathrm{g}$ and $\mathrm{e}$ are adjacent but $\mathrm{g} \notin \mathrm{F}$. Thus $\mathrm{g}$ and $\mathrm{e}$ are adjacent edges but $\mathrm{g} \notin \mathrm{F}-\{\mathrm{e}\}$ and $\mathrm{e} \notin \mathrm{F}-\{\mathrm{e}\}$, it implies that $\mathrm{F}-\{\mathrm{e}\}$ is not a strong edge cover of $\mathrm{G}$. Thus $\mathrm{F}$ is a minimal strong edge cover of $\mathrm{G}$.

Proposition 3.8. Let $G$ be a graph and $v \in V(G)$. Let $F$ be any strong edge cover of $G$ then there is at most one edge incident at $\mathrm{v}$ which is not in $\mathrm{F}$.

Proof. Suppose that there are two edges $\mathrm{vw}_{1}$ and $\mathrm{vw}_{2}$ which are not in $\mathrm{F}$. Thus, the edges $\mathrm{vw}_{1}$ and $\mathrm{vW}_{2}$ are adjacent but none of them is in $\mathrm{F}$, which contradicts the fact that $\mathrm{F}$ is a strong edge cover of $\mathrm{G}$.

Definition 3.9. [3] An edge H-dominating set with minimum cardinality is a minimum edge $\mathrm{H}$ dominating set. The cardinality of a minimum edge $\mathrm{H}$-dominating set is the edge H-domination number of $\mathrm{G}$, denoted by $\gamma_{H}^{\prime}(\mathrm{G})$.

Remark 3.10. (1) Every strong edge cover of the graph $\mathrm{G}$ with $\Delta(\mathrm{G}) \leq 2$ is an edge H-dominating set. That is every strong edge cover of a path graph and a cycle graph is an edge H-dominating set.

(2) By proposition 3.3, $\gamma_{H}^{\prime}(\mathrm{G}) \leq \alpha_{1}^{S}(\mathrm{G})$.

\section{VERTEX REMOVAL FROM THE GRAPH}

We consider the operation vertex removal from the graph. We assume that when a particular vertex $\mathrm{v}$ is removed from the graph $G$ then $G-v$ does not have isolated vertices. 
Example 4.1. Consider the graphs of the following figure, the graphs obtained after removal of a vertex from each graph of row 2 are given in row 1.

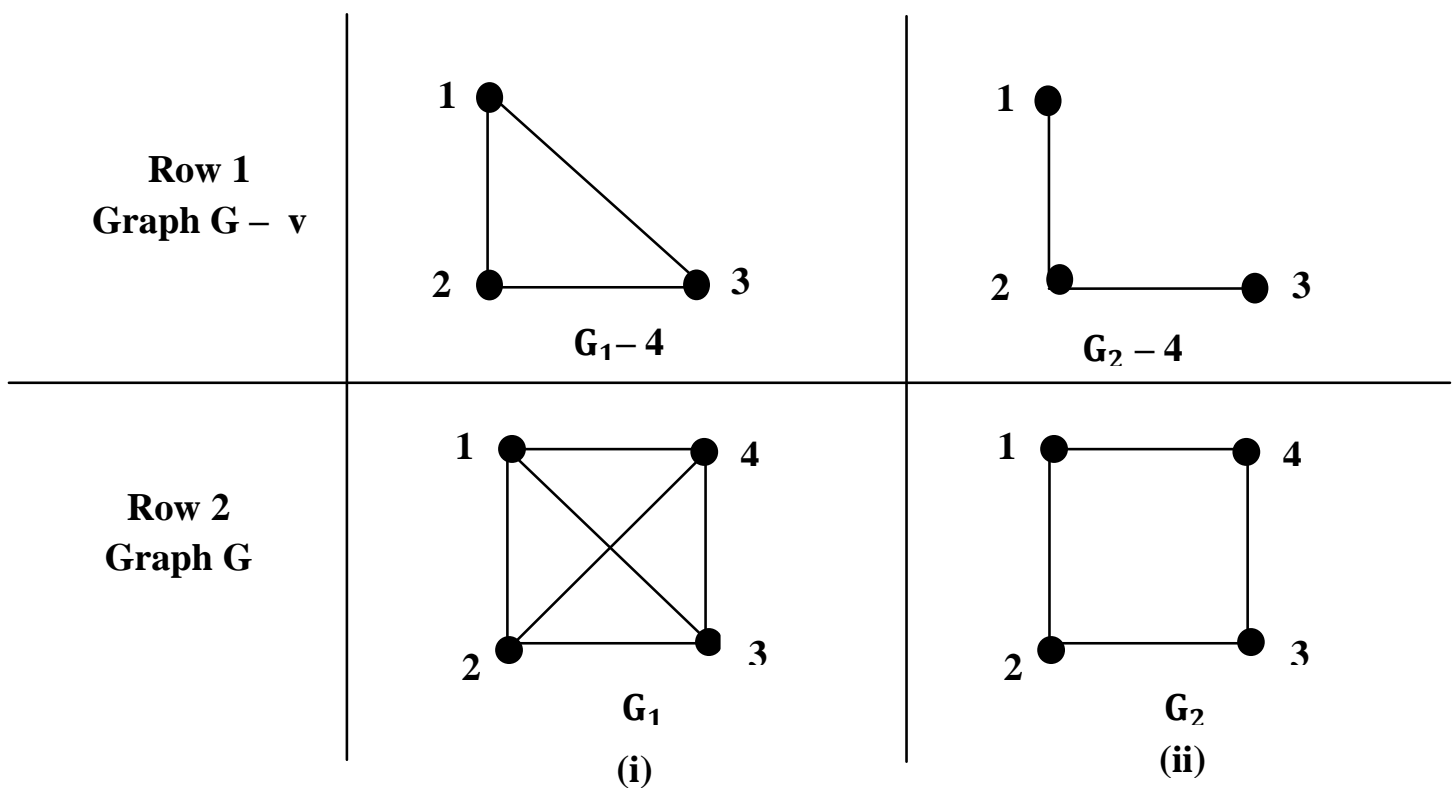

Figure 3. Graphs indicating vertex removal effect on strong edge cover

The effect of vertex removal on the strong edge covering number of the graph is indicated with the examples (i) and (ii) of figure 3.

(i) Consider the graph $\mathrm{G}_{1}$ ((i) of row 2). One of the minimum strong edge cover of this graph is $\{12$, $13,34,24\}$. The strong edge covering number of this graph is 4 . By removing any vertex (suppose vertex 4) from the graph, the strong edge covering number of the resultant (cycle) graph C3 ((i) of row 1) is 2 . Thus, the strong edge covering number of the graph decreases when a vertex is removed from the graph.

(ii) Consider the graph $\mathrm{G}_{2}$ ((ii) of row 2). The minimum strong edge covers of $\mathrm{G}_{2}$ are $\{12,34\}$ and $\{23,41\}$. The strong edge covering number of $\mathrm{G}_{2}$ is 2 . If any vertex (suppose vertex 4) is removed from $G_{2}$ then it gives a path graph ((ii) of row 1). The strong edge covering number of path graph $\mathrm{P}_{3}$ is also 2 . In this example, the strong edge covering number does not change when a vertex is removed from the graph.

In subsequent results, we will assume that $\mathrm{G}-\mathrm{v}$ does not have isolated vertices. The following theorem says that the strong edge covering number of the graph does not increase when a vertex is removed from the graph.

Theorem 4.2. Let $G$ be a graph and $v \in V(G)$ such that $G-v$ does not have any isolated vertex then $\alpha_{1}^{s}(\mathrm{G}-\mathrm{v}) \leq \alpha_{1}^{s}(\mathrm{G})$.

Proof. Let $\mathrm{F}$ be a minimum strong edge cover of $\mathrm{G}$. Consider any edge vu incident at v.

Case 1. If $\mathrm{vu} \notin \mathrm{F}$ then all the other edges incident at $\mathrm{u}$ are in $\mathrm{F}$.

Case 2. If $v u \in F$ then it is possible that there is some edge uw which is not in $F$. By proposition 3.8, the number of edges like uw can not be more than one. Now consider the set F. Remove all the edges vu which are in $\mathrm{F}$ and replace each edge vu by a unique edge uw different from vu (if required). After this operation, we get a new set of edges which we call $F_{1}$. Since each edge vu which was in $F$ is replaced by a unique edge uw which was not in $F$. Obviously, $F_{1}$ is a strong edge cover of $\mathrm{G}-\mathrm{v}$. Thus, $\alpha_{1}^{S}(\mathrm{G}-\mathrm{v}) \leq\left|\mathrm{F}_{1}\right| \leq|\mathrm{F}|=\alpha_{1}^{S}(\mathrm{G})$.

Theorem 4.3. Let $\mathrm{G}$ be a graph with $\delta(\mathrm{G}) \geq 3$ and $\mathrm{v} \in \mathrm{V}(\mathrm{G})$ then $\alpha_{1}^{s}(\mathrm{G}-\mathrm{v})<\alpha_{1}^{s}(\mathrm{G})$.

Proof. Let $F$ be a minimum strong edge cover of $G$ and $F_{1}$ be the set of edges obtained by removing all the edges which are incident at $v$ and which are in $F$. Obviously, $\left|F_{1}\right|<|F|$.

Let $\mathrm{vu}_{1}, \mathrm{vu}_{2}, \ldots, \mathrm{vu}_{\mathrm{k}}$ be all the edges which are incident at $\mathrm{v}$ and which are members of $\mathrm{F}$. For any $i, v u_{i} \in F$. Therefore, there is atmost one edge incident at $u_{i}$ which is not in $F_{1}$. If $v w$ is an 
edge which is not in $\mathrm{F}$ then also there is atmost one edge incident at $\mathrm{w}$ which is not in $\mathrm{F}_{1}$. Thus $\mathrm{F}_{1}$ is a strong edge cover of $\mathrm{G}-\mathrm{v}$. Thus, $\alpha_{1}^{S}(\mathrm{G}-\mathrm{v})<\alpha_{1}^{S}(\mathrm{G})$.

\section{EDGE REMOVAL FROM THE GRAPH}

Theorem 5.1. Let $\mathrm{G}$ be a graph with $\delta(\mathrm{G}) \geq 3$ and $\mathrm{e} \in \mathrm{E}(\mathrm{G})$ then $\alpha_{1}^{S}(\mathrm{G}-\mathrm{e}) \leq \alpha_{1}^{s}(\mathrm{G})$.

Proof. Let $F$ be a minimum strong edge cover of $G$ and e $\in E(G)$.

Case 1. Suppose that e $\notin F$.

Let $f_{1}$ and $f_{2}$ be two edges of $G-e$ which are adjacent in $G-$ e. Therefore $f_{1}$ and $f_{2}$ are adjacent in $G$ also. Since $F$ is a strong edge cover of $G, f_{1} \in F$ or $f_{2} \in F$. Therefore $F$ is a strong edge cover of $\mathrm{G}-\mathrm{e}$. Hence, $\alpha_{1}^{S}(\mathrm{G}-\mathrm{e}) \leq \alpha_{1}^{S}(\mathrm{G})$.

Case 2. Suppose that e $\in$ F.

Consider the set $F_{1}=F-\{e\}$. Let $f_{1}$ and $f_{2}$ be two edges of $G-e$ which are adjacent in $G-e$. Since $F$ is a strong edge cover of $G, f_{1} \in F$ or $f_{2} \in F$. Since $f_{1} \neq e$ and $f_{2} \neq e, f_{1} \in F_{1}$ or $f_{2} \in F_{1}$. Thus, $\mathrm{F}_{1}$ is a strong edge cover of $\mathrm{G}-\mathrm{e}$. Therefore, $\alpha_{1}^{S}(\mathrm{G}-\mathrm{e}) \leq\left|\mathrm{F}_{1}\right|<|\mathrm{F}|=\alpha_{1}^{S}(\mathrm{G})$.

Hence, from both the cases, $\alpha_{1}^{S}(\mathrm{G}-\mathrm{e}) \leq \alpha_{1}^{S}(\mathrm{G})$.

Now we prove the necessary and sufficient condition under which the strong edge covering number decreases when an edge is removed from the graph.

Theorem 5.2. Let $\mathrm{G}$ be a graph with $\delta(\mathrm{G}) \geq 3$ and $\mathrm{e} \in \mathrm{E}(\mathrm{G})$. The inequality $\alpha_{1}^{S}(\mathrm{G}-\mathrm{e})<\alpha_{1}^{S}(\mathrm{G})$ holds if and only if there is a minimum strong edge cover $F$ of $G$ such that $e \in F$.

Proof. Suppose that $F$ is a minimum strong edge cover of $G$ such that $e \in F$.

Let $\mathrm{F}_{1}=\mathrm{F}-\{\mathrm{e}\}$ then as proved in case 2 of theorem 5.1, $\mathrm{F}_{1}$ is a strong edge cover of $\mathrm{G}-\mathrm{e}$. Thus, $\alpha_{1}^{S}(\mathrm{G}-\mathrm{e}) \leq\left|\mathrm{F}_{1}\right|<|\mathrm{F}|=\alpha_{1}^{S}(\mathrm{G})$. That is, $\alpha_{1}^{S}(\mathrm{G}-\mathrm{e})<\alpha_{1}^{S}(\mathrm{G})$.

Conversely, suppose that $\alpha_{1}^{S}(\mathrm{G}-\mathrm{e})<\alpha_{1}^{S}(\mathrm{G})$.

Let $F_{1}$ be a minimum edge cover of $\mathrm{G}-\mathrm{e}$ then $\mathrm{F}_{1}$ cannot be an edge cover of $\mathrm{G}$ since $\alpha_{1}^{s}(\mathrm{G}-\mathrm{e})$ $<\alpha_{1}^{S}(\mathrm{G})$. Let $\mathrm{F}=\mathrm{F}_{1} \cup\{\mathrm{e}\}$ then $\mathrm{F}$ is an edge cover of $\mathrm{G}$ and also $\mathrm{e} \in \mathrm{F}$.

Corollary 5.3. Let $\mathrm{G}$ be a graph with $\delta(\mathrm{G}) \geq 3$ and e $\in \mathrm{E}(\mathrm{G})$. The equality $\alpha_{1}^{s}(\mathrm{G}-\mathrm{e})=\alpha_{1}^{s}(\mathrm{G})$ holds if and only if e $\notin \mathrm{F}$ for any minimum strong edge cover $\mathrm{F}$ of $\mathrm{G}$.

\section{CONCLusions}

The results written in the paper are based on the parameters related to the edges of the graph. The graph operations like vertex removal and edge removal are considered. In particularly, the effect of these operations on the strong edge cover of the graph is observed.

\section{REFERENCES}

[1] Alewyn P. Burger, Michael A. Henning, Jan H. van Vuuren, Vertex covers and secure domination in graphs, Quaestiones Mathematicae, (31)(2008), 163 - 171.

[2] T. W. Haynes, S. T. Hedetniemi and P. J. Slater, Fundamental of Domination in Graphs, Marcel Dekker, Inc., New York, 1998.

[3] B. M. Kakrecha, Ph. D. Thesis, Saurashtra University, Rajkot, 2017.

[4] D. K. Thakkar and B. M. Kakrecha, About the Edge Covers of the Graph. International Journal of Scientific and Innovative Mathematical Research, 5(1)(2017), 1 - 7.

[5] D. K. Thakkar and B. M. Kakrecha, An algorithmic approach to obtain an edge H-dominating set of the graph, Bulletin of Mathematics and Statistics Research, 4(4)(2016), $164-169$.

[6] D. K. Thakkar and B. M. Kakrecha, Edge H-domination in Graphs, Submitted for possible publication. 

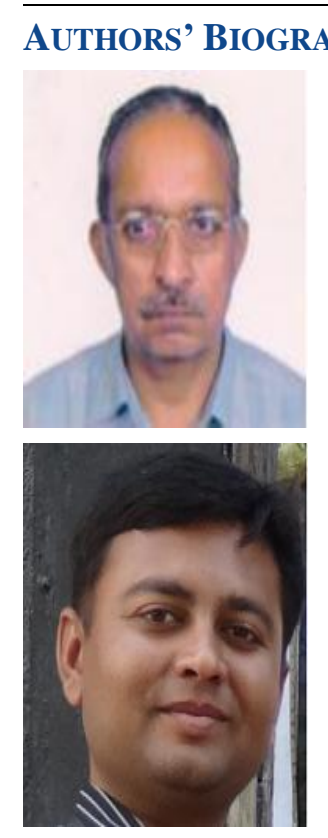

D. K. Thakkar, is a Professor and Head at the Department of Mathematics, Saurashtra University, Rajkot, Gujarat (India). His areas of interest are Graph Theory, Topology and Discrete Mathematics. He has published over 75 research papers in various journals.

Bhavesh M. Kakrecha has obtained his M. Sc. degree and M. Phil. degree in Mathematics from Department of Mathematics, Saurashtra University, Rajkot, Gujarat (India) in 2009 and 2010 respectively. At present, he is working as an Assistant Professor in Mathematics at Lukhdhirji Engineering College, Morbi, Gujarat (India). He has obtained his Ph. D. degree in Mathematics from Department of Mathematics, Saurashtra University, Rajkot in 2017 under the supervision of Dr. D. K. Thakkar.

Citation: D. K. Thakkar, B. M. Kakrecha, "Strong Edge Cover of the Graph ", International Journal of Scientific and Innovative Mathematical Research, vol. 5, no. 10, p. 9-14, 2017., http://dx.doi.org/10.20431/ 2347-3142.0510002

Copyright: () 2017 Authors. This is an open-access article distributed under the terms of the Creative Commons Attribution License, which permits unrestricted use, distribution, and reproduction in any medium, provided the original author and source are credited. 